
Not for reproduction, distribution or commercial use.

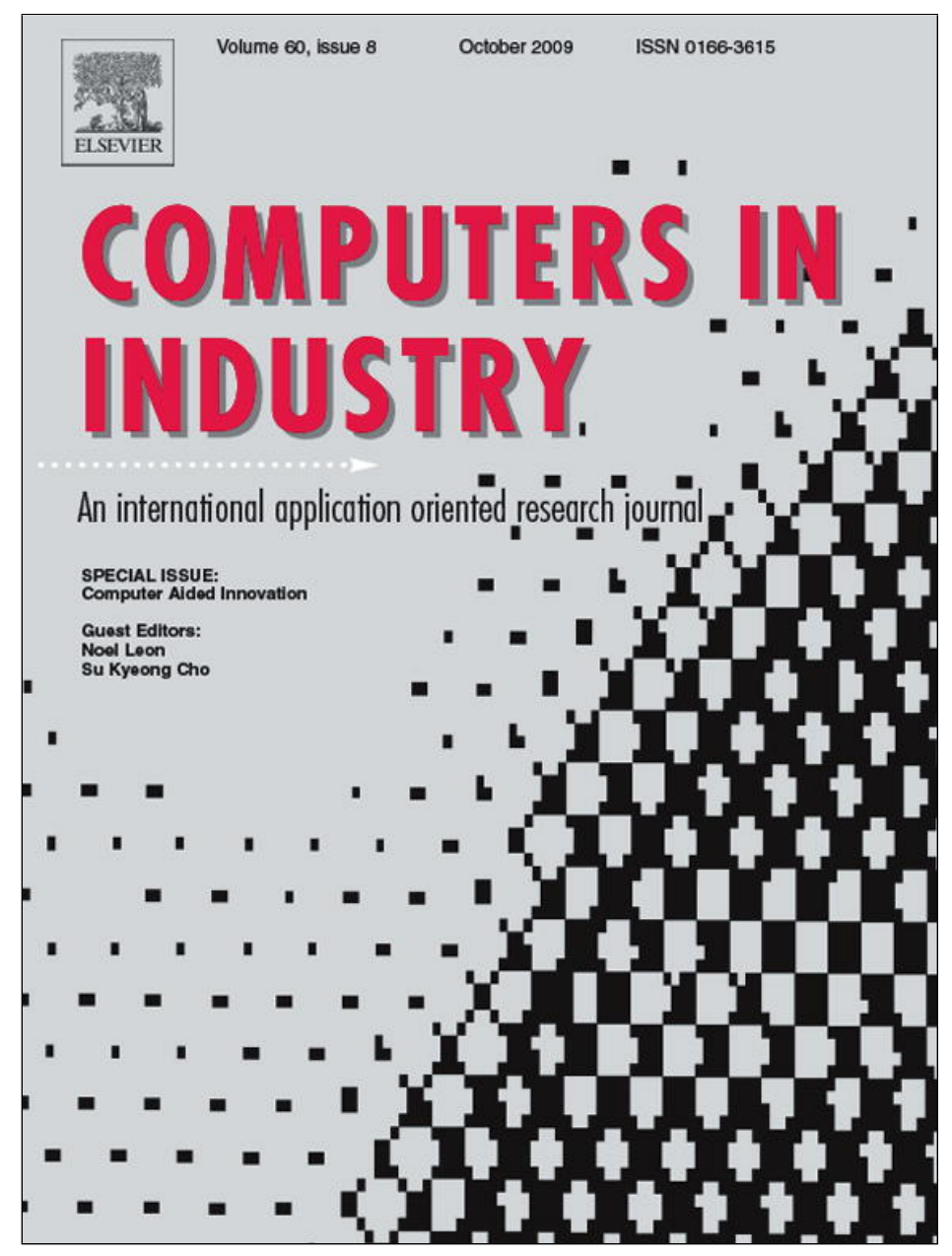

This article appeared in a journal published by Elsevier. The attached copy is furnished to the author for internal non-commercial research and education use, including for instruction at the authors institution and sharing with colleagues.

Other uses, including reproduction and distribution, or selling or licensing copies, or posting to personal, institutional or third party websites are prohibited.

In most cases authors are permitted to post their version of the article (e.g. in Word or Tex form) to their personal website or institutional repository. Authors requiring further information regarding Elsevier's archiving and manuscript policies are encouraged to visit: 


\title{
Integrated Computer-Aided Innovation: The PROSIT approach
}

\author{
Umberto Cugini $^{\text {a }}$, Gaetano Cascini ${ }^{\mathrm{b}, *}$, Maurizio Muzzupappa ${ }^{\mathrm{c}}$, Vincenzo Nigrelli ${ }^{\mathrm{d}}$ \\ a Politecnico di Milano, Dipartimento di Meccanica, Milano, Italy \\ ${ }^{\mathrm{b}}$ Università di Firenze, Dip. di Meccanica e Tecnlogie Ind.li, Florence, Italy \\ c Università della Calabria, Dip. di Meccanica, Cosenza, Italy \\ ${ }^{\mathrm{d}}$ Università di Palermo, Dip. di Meccanica, Palermo, Italy
}

\section{A R T I C L E I N F O}

\section{Article history:}

Available online 9 July 2009

\section{Keywords:}

Computer-Aided Innovation

Knowledge-Based Engineering

Optimization systems

Product Lifecycle Management

\begin{abstract}
A B S T R A C T
The paper presents a methodology aimed at the improvement of the product development cycle through the integration of Computer-Aided Innovation (CAI) with Optimization and PLM systems. The interoperability of these tools is obtained through the adoption of Optimization systems as a bridging element between CAI and PLM systems. This methodology was developed within the PROSIT project (http://www.kaemart.it/prosit).

The paper describes the main issues related to the integration of these complementary instruments and the solutions proposed by the authors. More specifically, the main idea of the PROSIT project to link CAI and Optimization systems is the adoption of the latter tools not just to generate optimized solutions, but also as a design analysis tool, capable to outline critical aspects of a mechanical component in terms of conflicting design requirements or parameters. CAI systems are then applied to overcome the contradictory requirements. The second step, i.e. the integration between Optimization and PLM systems, has been obtained through the development of Knowledge-Based (KB) tools to support designer's activities. More in details, they provide means to analyze and extrapolate useful geometrical information from the results provided by the optimizer, as well as semi-automatic modelling features for some specific geometries. A detailed example related to the design of a plastic wheel for light motoscooters clarifies the whole procedure. The paper integrates, extends and updates topics presented in Cugini et al., Barbieri et al. and Cascini et al. [U. Cugini, G. Cascini, M. Ugolotti, Enhancing interoperability in the design process-the PROSIT approach, in: Proceedings of the 2nd IFIP Working Conference on Computer-Aided Innovation, Brighton (MI), USA, October 8-9, 2007, published on Trends in ComputerAided Innovation, Springer, ISBN 978-0-387-75455-0, pp. 189-200; L. Barbieri, F. Bruno, M. Muzzupappa, U. Cugini, Design automation tools as a support for knowledge management in topology optimization, in: Proceedings of the ASME 2008 International Design Engineering Technical Conferences \& Computers and Information in Engineering Conference (IDETC/CIE 2008), Brooklyn, New York, USA, August 3-6, 2008; L. Barbieri, F. Bruno, M. Muzzupappa, U. Cugini, Guidelines for an efficient integration of topological optimization tools in the product development process, in: Third International Conference on Design Computing and Cognition, Atlanta, USA, June 23-25, 2008; G. Cascini, P. Rissone, F. Rotini, From design optimization systems to geometrical contradictions, in: Proceedings of the 7th ETRIA TRIZ Future Conference, Frankfurt, Germany, November 6-8, 2007].
\end{abstract}

(c) 2009 Elsevier B.V. All rights reserved.

\section{Introduction}

Market competitiveness through innovation is the common strategy adopted by leading companies of developed countries, even if the concept of innovation itself is very often abused and misused. Certainly, in order to release novel and valuable products, a crucial aspect for a company is the efficiency of its product development

\footnotetext{
* Corresponding author.

E-mail addresses: umberto.cugini@polimi.it (U. Cugini),

gaetano.cascini@unifi.it (G. Cascini), muzzupappa@unical.it (M. Muzzupappa), nigrelli@dima.unipa.it (V. Nigrelli).
}

cycle from the so-called fuzzy front end characterizing the problem setting phase to the detailed design phase. In other terms companies have to implement not just means for generating new ideas with a systematic approach, but also an integrated environment where effective ideas are efficiently converted into products.

Computer applications play a relevant role for increasing the efficiency of the whole process. However, up to now systematic innovation methodologies like TRIZ are still poorly integrated with product embodiment means [5]. This is because:

- the CAD/CAE systems are not conceived for supporting the designer in conceptual design activities as always imply a complete, well defined geometric model, 
- at the same time the outputs of a TRIZ problem solving tool (such as inventive/separation principles, standard solutions, etc.) can hardly be translated into a modification of a CAD model and the only opportunity is to restart the modelling process.

A few preliminary experiments to integrate TRIZ principles within CAD systems have been attempted with promising, but still not satisfactory, results [6-8]. Besides, a small consortium of Italian Universities has carried out the PROSIT project (http:// www.kaemart.it/prosit), "From Systematic Innovation to Integrated Product Development", with the aim of bridging systematic innovation practices and Computer-Aided Innovation (CAI) tools with Product Lifecycle Management (PLM) systems, by means of Design Optimization tools [1].

The basic starting point is that in any design process designers have to address, among the others, three subsequent interconnected tasks:

- problem setting (precisely formulate the right question);

- define the correct/optimal architectural/morphological answer;

- finalize the best solution taking into account technical/engineering constraints.

To accomplish these tasks, designers have at their disposal different dedicated approaches and tools. The goal of PROSIT project was to demonstrate that is possible to define a coherent and integrated approach leveraging on available theories, methods and tools as illustrated in Fig. 1.

Innovation and optimization are usually perceived as conflicting activities. In this project, topology and shape generation capabilities of modern Design Optimization technologies are adopted as a means to speed-up the embodiment of innovative concepts, but also as a way to support the designer in the analysis of conflicting requirements for an easier implementation of TRIZ instruments for conceptual design.

In this paper the use of Design Optimization tools is proposed for identifying the physical contradictions underlying a mechanical system, i.e. the generalized problem model according to traditional TRIZ theory; then, TRIZ general models of solutions (i.e. inventive/separation principles and geometrical effects) are converted into new optimization problems in order to implement a novel solution. Moreover the PROSIT project has allowed the development of some KB user interfaces to process the outputs of an Optimization system with the aim of speeding up their translation into a solid model.

Section 2 reports a brief survey of the relevant State of the Art in order to highlight opportunities and interoperability limits of actual CAI, Optimization and PLM systems; Section 3 details the procedure developed within the PROSIT project and describes the main features of the guidelines and instruments implemented so far. Section 4 shows an exemplary application related to the design of a plastic wheel, while the conclusions are briefly presented in Section 5 .

\section{Related art}

Several Computer-Aided Innovation systems have been proposed recently, with promising results especially when adopted with a proper methodological background. In [9] a detailed excursus of TRIZ software evolution is reported with a specific attention to the original research of the first community in the former Soviet Union. Both the first attempts to build computerbased applications supporting a TRIZ problem solving process and the most recent software products comprising TRIZ-based modules still require the users to be familiar with Classical TRIZ at each stage of formulation and modelling of the problem to be solved, as well as for interpreting the resulting models of solution. Therefore, it can be concluded that the actual paradigm of these CAI applications constitutes the first limit to their diffusion, due to the need of an adequate theoretical background to get the expected benefits.

Besides, Optimization tools and PLM/EKM systems have already demonstrated their relevant potentialities to increase the effectiveness of specific design activities. Nevertheless, the potential of a smooth and effective integration of all these computer-based systems has not been fully exploited. This is primarily due to firstly a poor integration between different applications, and secondly by the difficulty to fully understand how to take advantages of these tools and how to effectively use them in the context of the Product Development Process. Indeed, it has been estimated that the United States industry spends billions of dollars because of poor interoperability between Computer-Aided Engineering software tools [10].

A full integration of these technologies is still far to be reached [11], and big efforts are required to set up successful collaborations and to push companies to focus their attention on the adoption of

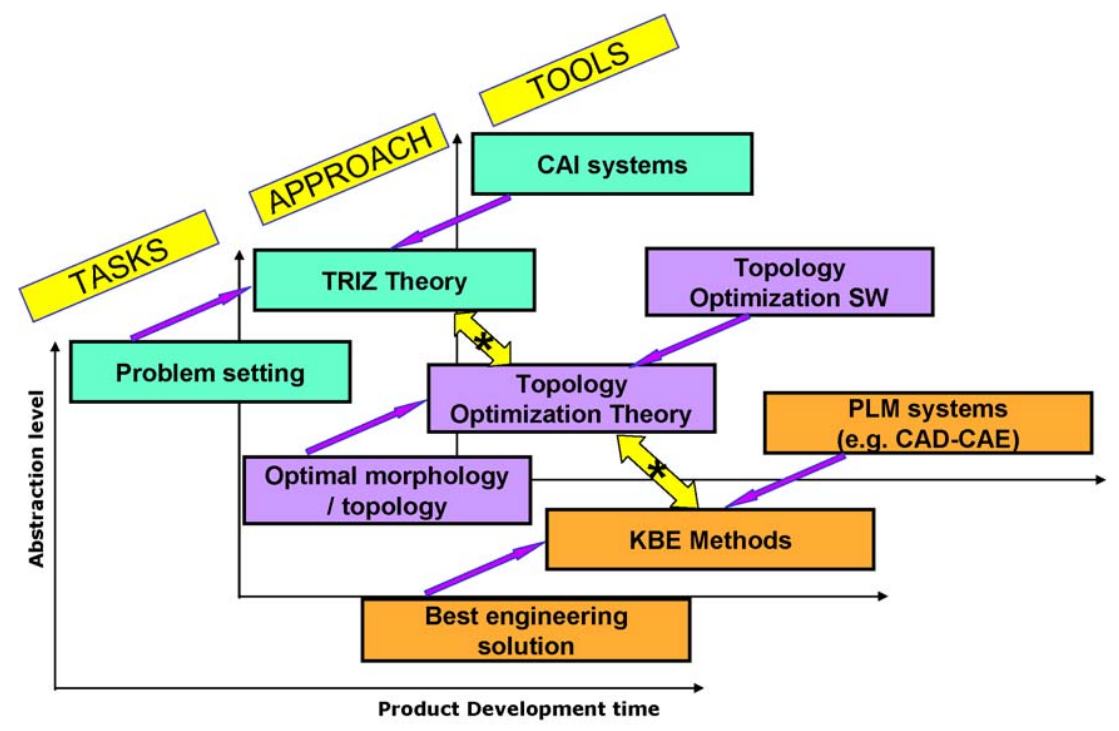

Fig. 1. Layered representation of approach/methods and tools supporting the problem solving tasks of a Product Development Process. 
new organizational paradigms to better coordinate the design activity in such a context. The capability to support in a more integrated way all the stages of the Product Development Process (PDP) will be one of the most important competitive factors for these systems in the next years. In a concurrent engineering view, in fact, it is required that the phases of conceptual design, optimization and detailed design would be integrated as far as possible. In order to reduce development time and increase activities' effectiveness, design and Product Development Process have to be considered as a continuous iteration among these phases.

\subsection{Computer-Aided Innovation vs. Design Optimization}

Designing by optimization techniques means translating a design task into a mathematical problem with the following basic entities:

- An objective function, i.e. the performance of the system that the designer wants to reach or to improve;

- A set of design variables, i.e. the parameters of the system affecting the objective function;

- A set of loading conditions and constraints representing the requirements the system has to satisfy.

The optimization algorithm finds the value of the design variables that minimizes, maximizes, or, in general, "improves" the objective function while satisfying the constraints. The use of computers for Design Optimization is rather common in several fields since 1980s. Furthermore, during the last years new optimization tools have been developed to solve specific design problems [12,13]. In the following, the main features of these techniques will be summarized.

In a shape optimization process the outer boundary of the structure is modified according to the optimization task. The shape of the structure, modelled through the finite element method, is modified by the node locations: the optimization algorithm, according to the loads and boundary conditions applied to the FE model, changes the coordinates of the nodes which are defined as design variables. The result of the optimization cycle is a deformed geometry of the starting shape structure.

The size optimization is a special type of parametrical optimization in which the design variables are represented by some properties of structural elements such as shell thickness, beam cross-sectional properties, spring stiffness, mass, etc. During the optimization process, these parameters are modified by the algorithm until the expected goal is reached.

Topology optimization is a technique that determines the optimal material distribution within a given design space, by modifying the apparent material density considered as a design variable. The design domain is subdivided into finite elements and the optimization algorithm alters the material distribution within the design space at each iteration, according to the objective and constraints defined by the user. The external surfaces defined as "functional" by the user, are kept out from the optimization process and considered as "frozen" areas by the algorithm.

Topography optimization is an advanced form of shape optimization in which a distribution of ribs and pattern reinforcements is generated on a specific design region. The approach in topography optimization is similar to the approach used in topology optimization, but shape variables (node coordinates) are used instead of density variables. The large number of shape variables allows the user to create any reinforcement pattern within the design domain.

Moreover, manufacturing constraints may be set in order to take into account of the requirements related to the manufacturing process. Sliding planes and preferred draw directions may be imposed for moulded, tooled and stamped parts as well as minimum or maximum size of the structural elements (i.e. ribs and wall thicknesses).

However, since the design process has multidisciplinary characteristics, improving one performance of a system may result in degrading another. This kind of conflicts cannot be solved using Design Optimization because these techniques are able to focus the design task only to one specific performance to be improved. More precisely, Design Optimization tools allow management of multiple goals just by defining complex objective functions where a weight must be assigned to each specific goal [14]. Thus, the best compromise solution is generated on the base of an initial assumption made by the designer about the relative importance of the requirements, without taking account of the reciprocal interactions.

Besides, the logic behind several CAI systems is mostly related to the TRIZ theory, i.e. to the refusal of trade-offs; thus, they are apparently in conflict with the logic of optimization, seen as minimization of negative issues within a given set of constraints. These antithetical characteristics of ComputerAided Innovation and Design Optimization require the definition of a new integrated design environment capable not only to explore values of attributes (decision variables) within a given design space, but also to evolve the quantity and the quality of these attributes, i.e. when changes in the representation space occur.

According to the design flow proposed within the PROSIT project, the role of the optimization systems is to explore the design space, while the creative step is demanded to the redefinition of the design space and/or the optimization objectives and constraints made through the implementation of the TRIZ guidelines. More specifically, the traditional approach to optimization involves the application of a complete system of constraints and loads to the geometry for describing all the design requirements.

Since this "optimal", i.e. "best compromise" solution is unnecessarily satisfying, it is often useful, before moving towards the detailed definition of the product architecture, to re-discuss already made assumptions, in order to obtain a solution, which better satisfies general system objectives. Based on these considerations, the authors propose to perform a set of mono-objective optimization tasks in order to put in evidence conflicts among geometrical elements of the system under analysis.

\subsection{KBE Systems and Design Optimization integration}

The problem of integrating topological optimization tools in Product Development Process (PDP) is becoming more and more urgent since nowadays they are widely employed in several engineering fields (civil, aeronautics, aerospace and automotive). The use of such systems is critical because when a virtual model of the product undergoes an optimization cycle, the connected information is lost. The result is that the optimized model takes into consideration neither functional and technological features, nor rules and features associated to the various parts of the product (this is due to the output of topological optimizers that is a voxel model). In practice, the geometry can be useless because the optimized model:

- may not be manufacturable or its manufacturing costs may be very high;

- violates design constraints, standards or functional aspects;

- turns out to be very complex to rebuild in terms of functional and technological features. 
In all these cases, the designer has to model the geometry again interpreting the results obtained by the optimization process, and considering standards and manufacturing features and constraints [15]. During the phase of feature-based redesign, the modules of feature recognition are the only tools offered by PLM systems as a support for the user. At present, only few PLM systems are able to offer the advantages of feature recognition tools. Among others, the most widespread are feature recognition 1 of Catia, Holemaking of UG/NX, Feature Recogniser of SolidEdge and Feature Works of Solid Works. On the other hand, in literature, the present researches recommend the use of genetic algorithms and neural networks, in order to expand and improve the feature libraries $[16,17]$. However, these researches deal with few features to recognize and with 2D or 2D1/2 shapes (obtained by a projection of a 2D shape into 3rd dimension) and never with complex geometry [18-20].

In literature, several researchers have faced the optimization problem through, either classic approaches of shape recognition, or design by feature. Others have tried to introduce optimization into the phase of conceptual design with the adoption of hybrid CAD/ FEM systems. In the above-mentioned researches, structural characteristics, calculated a priori, are assigned to each component. Until now, these isolated attempts have not lead to consolidated solutions. In conclusion, feature recognition techniques are not able, at the moment, to support the post-processing of Topology Optimization results. This means that it is necessary to develop specific design methodologies that describe some formalized procedures, needed to implement a systematic introduction of topological optimization tools in PDP.

In conclusion, such solutions are not able to significantly solve the problem in question. In fact, such modules were not thought up for geometrically complex models and represented through voxel. The result of the optimization is subject to the designer's evaluation (usually through a visual analysis of the model).

\section{Methodology}

According to the diagram of Fig. 1, the PROSIT project aims at bridging three different classes of product development methods and systems, CAI and Optimization systems from one side, Optimization systems and PLM/EKM tools to the other.

\subsection{From optimization analysis to geometrical contradictions identification and solution}

The rationale behind the adoption of Optimization systems as a means for design analysis is the following:

- defining a single multi-goal optimization problem leads to a compromise solution;

- besides, defining $\mathrm{N}$ complementary mono-goal optimization problems, each with specific boundary conditions, leads to $\mathrm{N}$ different solutions;

- these solutions can be conflicting and this is the key to find contradictions.

According to this statement, the PROSIT design flow is structured as depicted in Fig. 2. The process starts with the definition of a multi-objective optimization analysis; if the results satisfy the whole set of constraints and requirements the designer can proceed towards the detailed design of the product. Besides, if the output of the multi-goal optimization does not fit the product specifications, a set of single-goal optimization tasks, each representing a specific operating condition and/or a given design requirement, must be defined.

A consequence of the lack of satisfactory solutions to the multiobjective optimization analysis is that the different single-goal optimization tasks lead to conflicting geometries, thus the system must be further investigated in order to extract the "geometrical contradictions" a subclass of physical contradiction proposed by

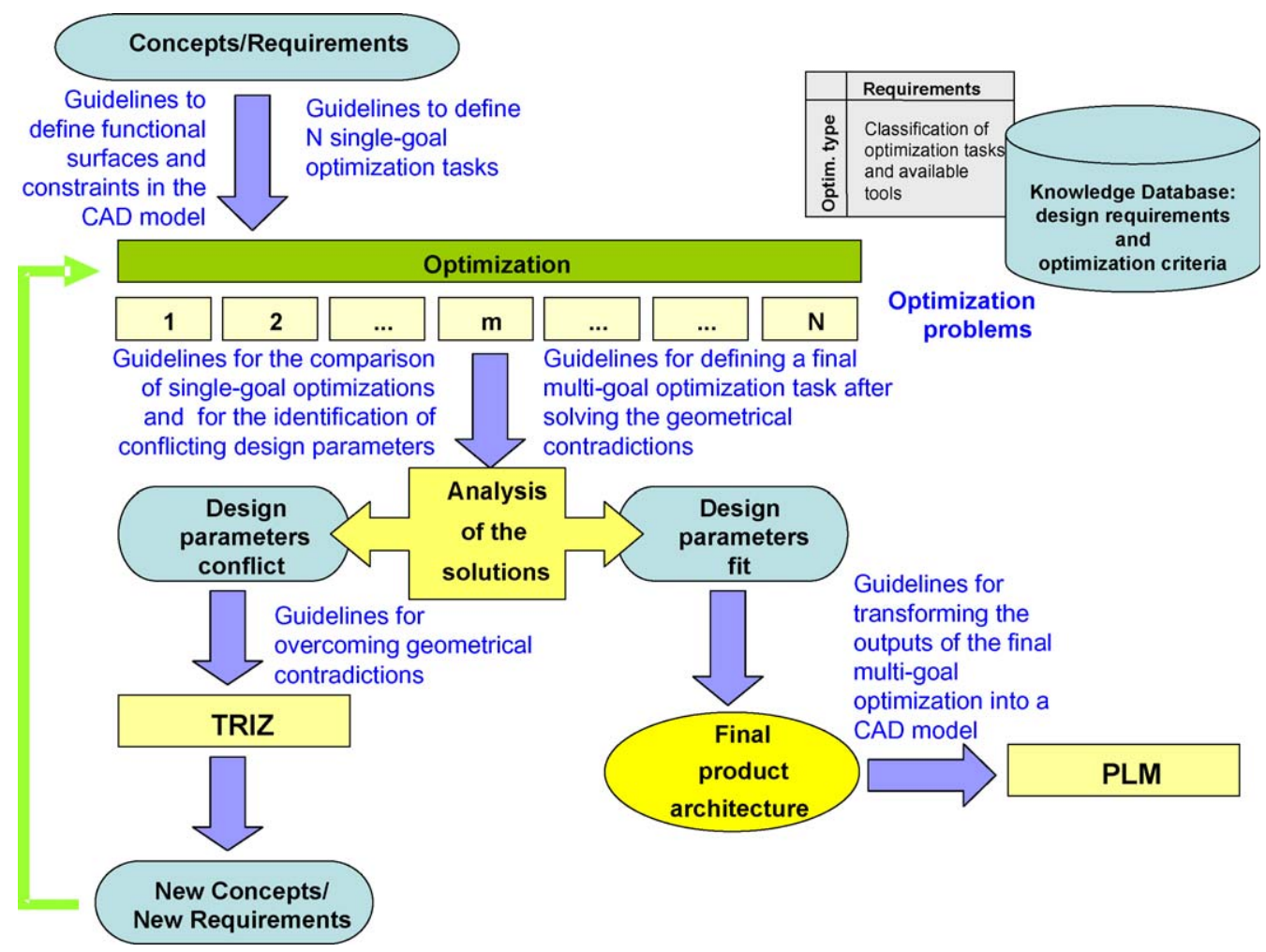

Fig. 2. Design flow according to the PROSIT approach. 


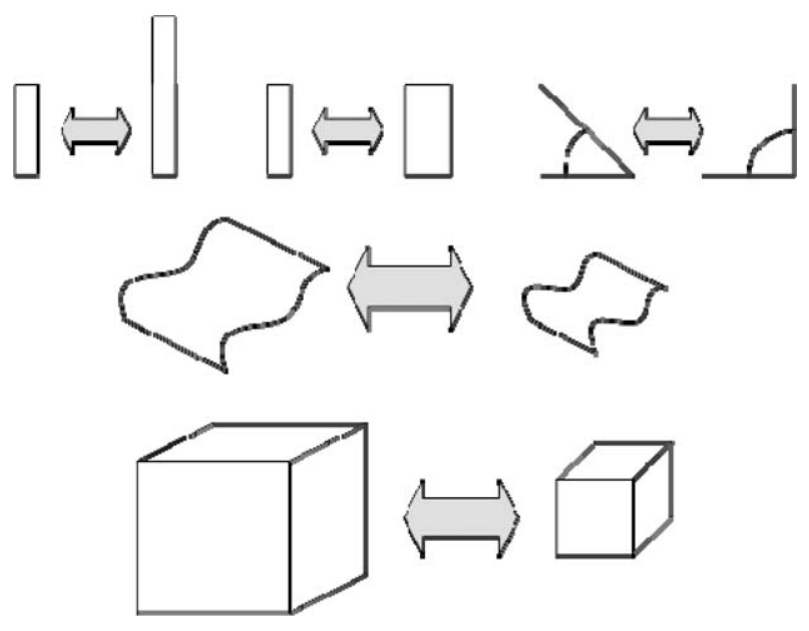

Fig. 3. Exemplary representations of size contradictions: 1D (above), 2D (middle) and $3 \mathrm{D}$ (below).

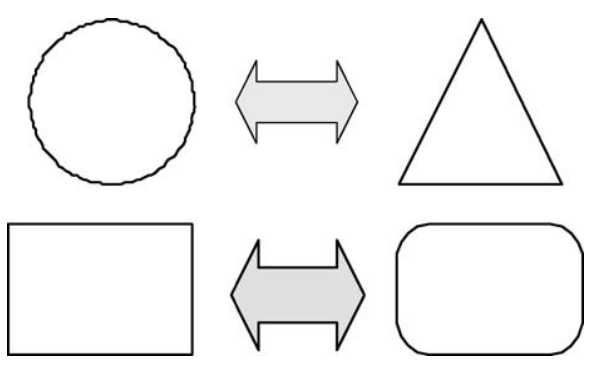

Fig. 4. Exemplary representations of shape contradictions: system level (above) and subsystem level (below).

the authors [4]. More specifically, according to the differences between the results of the single-goal optimization tasks and to the nature of the conflicting design parameters, the geometrical contradictions can be classified into:

- Size contradictions: a dimensional parameter of the Technical System (TS) should be big and should be small according to two or more different mono-goal optimization tasks. Three different sub-classes can be defined: 1D, 2D, 3D (Fig. 3).

- Shape contradictions: an element or a detail should assume different forms, e.g. sharp vs. rounded details, circular and polygonal (Fig. 4).

- Topological contradictions: an element or a detail should assume different topologies (material distributions, e.g. monolithic and segmented) and/or orientations (e.g. horizontal and vertical Fig. 5).

Basically the methodology guides the designer according to the classical TRIZ approach. The application of TRIZ instruments allows, in synthesis to:
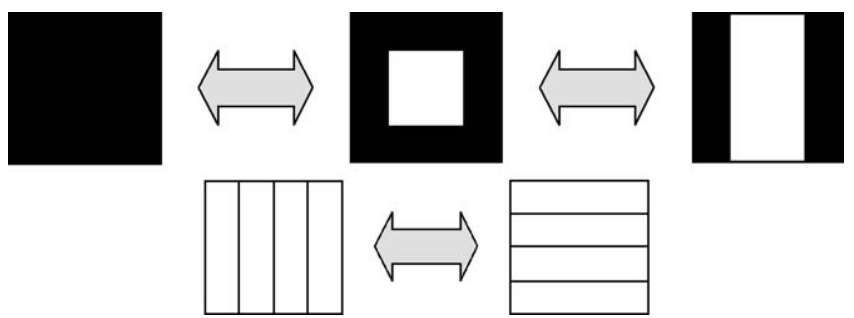

Fig. 5. Exemplary topological contradictions: different material distributions (above) or different position/orientation (below).

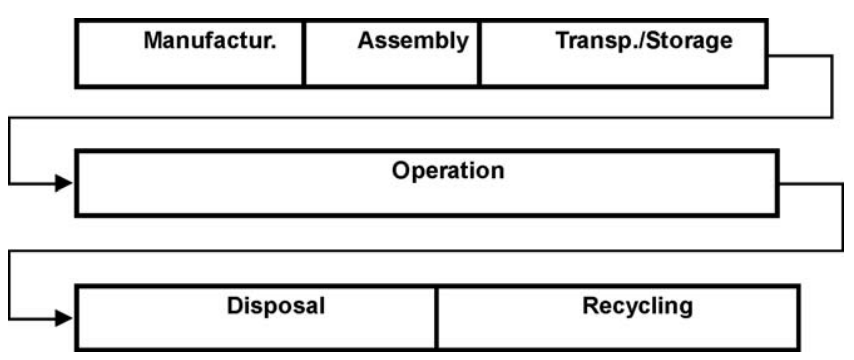

Fig. 6. Life phases of a TS taken into account for contradictions classification.

- identify the operational zone within the design space where the geometrical contradiction resides,

- check if contradictory requirements for the design parameters co-exist in the operational zone and time,

- check if those contradictory requirements co-exist under any condition and evaluate the opportunity to overcome them by means of a separation strategy or through a transition to subsystem elements or to its environment (i.e. ARIZ step 5.3, principles for overcoming physical contradictions, third class of the Inventive Standards).

In order to identify the most suitable direction to overcome the geometrical contradiction, it is useful to take into account also further information related to the differences between the conflicting mono-goal optimization problems:

- the life phase of the Technical System (TS) to which each optimization problem refers to (Fig. 6);

- the functional role of the geometrical elements where the conflict resides (Fig. 7), on the base of the classical TRIZ smallmodel constituted by a Tool, a Transmission, a Supply and a Control.

The research has been carried out by analyzing a 100 inventive solutions based on a geometrical evolution of the system, extracted from the authors' experience (a dozen of real case studies) and a higher number of patents identified through geometry-related terms. Such a set of selected geometrical solutions has been analyzed in terms of type of contradiction, maturity level of the product, Su-Field model representing its functional interactions, Geometrical Effects and Inventive Principles associated to the inventive step from the previous existing geometry to the invented solution. The inductive approach has been complemented with a deduction-based reasoning in order to organize the emerging correlations.

As a result, a pointer to the most suitable Inventive Principle or Geometrical Effect to overcome geometrical contradictions has been built and specific guidelines to update the boundary conditions of the Optimization system are provided.

A few exemplary deductions will be reported here after, while a more comprehensive description is demanded to a next publication. The format of these associations geometrical contradictionsolution path has been defined with the perspective of generating a Knowledge-driven user interface within the PROSIT software platform:

- If the geometrical contradiction involves both the operation and another stage of the product life (e.g. manufacturing and transportation), it is clear that separation in time strategies are conceptually feasible. In order to have a Technical System (TS) assuming different configurations/behaviours in different stages of its life, a typical solution principle is, for example, dynamization (TRIZ Inventive Principle IP\#15). 


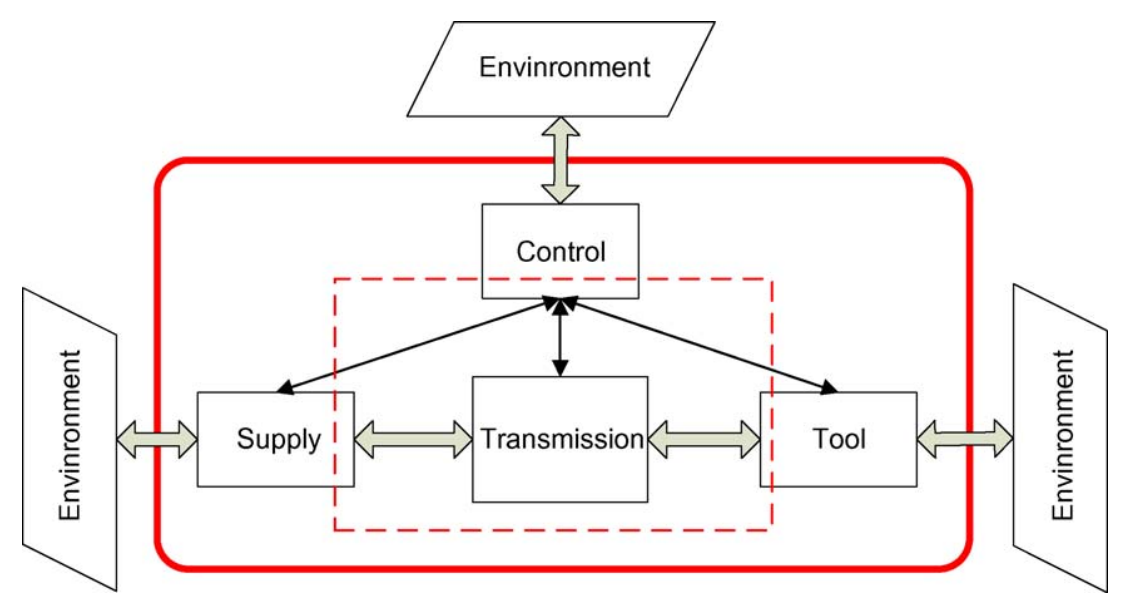

Fig. 7. Elementary functional model of a TS: the thick continuous line delimits the TS itself, the dashed line represents the portion of TS subjected to geometrical optimization in a typical Geometrical Optimization task (functional surfaces are not modified by Optimization Design tools).

- If the geometrical contradiction appears due to alternative requirements and/or loading conditions during the operation phase, a separation in time strategy means that the TS may assume different configurations. It is clear that such a solution is limited by the speed of the processes involved; besides, it is much easier to tune the behaviour of the TS according to slower processes (day/night, summer/winter, etc.).

- A geometrical contradiction belonging to the operation phase, such that the process is too fast to perform a separation in time or submitted to independent loading conditions (e.g. wind and/or snow) should be approached with a separation in space strategy (IP\#1 segmentation, IP\#17 another dimension, IP\#4 asymmetry, IP\#3 local quality, IP\#7 nested dolls, etc.). Since in the embodiment phase it is preferable to avoid major changes in the adjacent components, a separation in space is the best option if the geometrical contradiction is located in the functional transmission of the TS, i.e. the portion of the design space where the Optimization software tool is allowed to introduce modifications (the dashed box in Fig. 7). In this case, according to the dataset analyzed in this study, the type of geometrical contradiction (size-shape-topological) assumes a relevant role to point to a suitable solution path.

- If the geometrical contradiction is located outside the transmission, i.e. it involves the functional surfaces of the design volume, it is requested a change in the way the main useful function is delivered. It is worth reminding that this study is dedicated to the embodiment phase of product development, therefore the physical/chemical principle adopted to perform the main useful function should not be changed. Besides, it can be changed the way the functional flow of material/energy/information is introduced in the TS or is applied by the TS to the target of the action. Here a direct link to Vikentiev's pointer to Geometrical Effects [21,22] can be created. If the contradiction resides in the tool of the TS, the function associated to the tool should be adopted to enter in the Vikentiev's pointer to Geometrical Effects, while if the contradiction is related to the supply of the TS, it means that the TS is the receiver of a function to be assumed as the pointer input.

- In any case, if the geometrical contradiction covers two or more functional portions of the TS, i.e. two or more among tool, transmission, supply and control, a separation in space strategy can be applied, by assigning different values in different regions to the conflicting geometrical features/parameters.

- When the geometrical contradiction involves two different stages of life of the TS, for example manufacturing and operation, it is still useful to consider the operational space where the contradiction occur as an element of a supply-transmission-tool chain, in order to focus the attention on the portion of the design volume where a modification of the representation space is required. Such a situation is further clarified in the exemplary application of Section 4

Closing the loop, as a result of this activity, a new set of optimization problems can be identified and can be solved making use of the optimization tools. In other words, the TRIZ principles
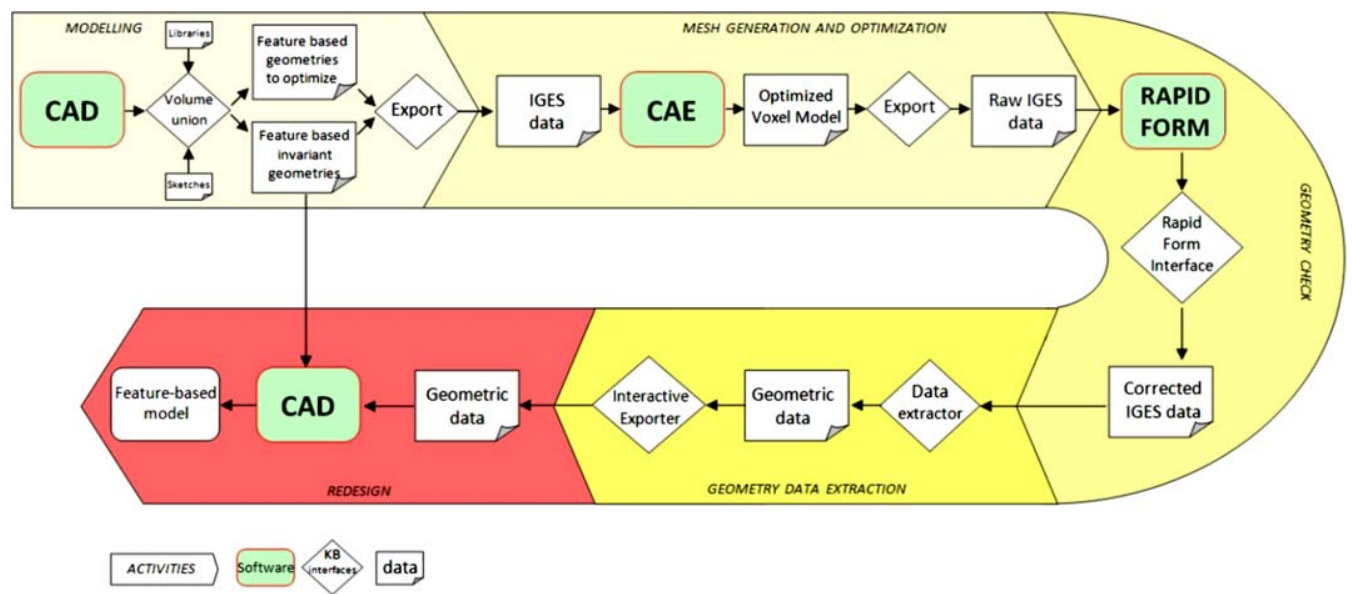

Fig. 8. Scheme of methodology and implemented tools. 


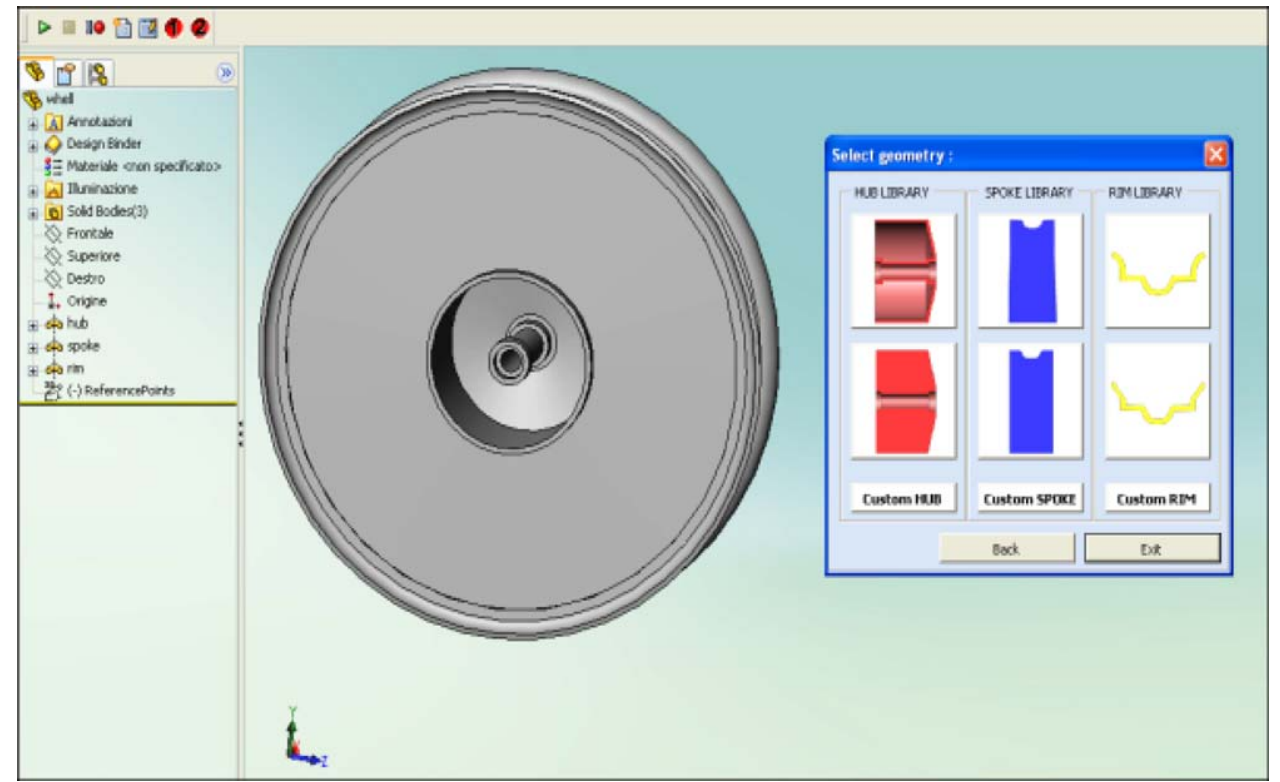

Fig. 9. Realization of a CAD model through the union of volumes belonging to a library.

are used to redefine the design volume, the functional surfaces and/or the optimization constraints so that the conflict between the design parameters disappears. This procedure has to be iterated until optimization process' results converge, i.e. the geometries generated by the different single-goal optimization tasks fit each other.

\subsection{From Optimization systems to PLM}

As regards the interoperability between Optimization systems (OSs) and PLM, the PROSIT projects has proposed the implementation of three KB custom user interfaces in order to automate, support and simplify the remodelling phase. Moreover, a preliminary definition of some guidelines to lead the engineer during the translation of topological optimization results into a technological feature-based geometry [2,3]. The steps of such methodology and all the tools implemented to support it are shown in Fig. 8.

The first step of the methodology is the preparation of the model to be optimized. It has been developed a first KB interface, able to model the component in a CAD environment, by joining standardized geometries, realized according to the case study or taken from custom libraries (Fig. 9). By using the interface, such geometries must successively be exported to a CAE environment,
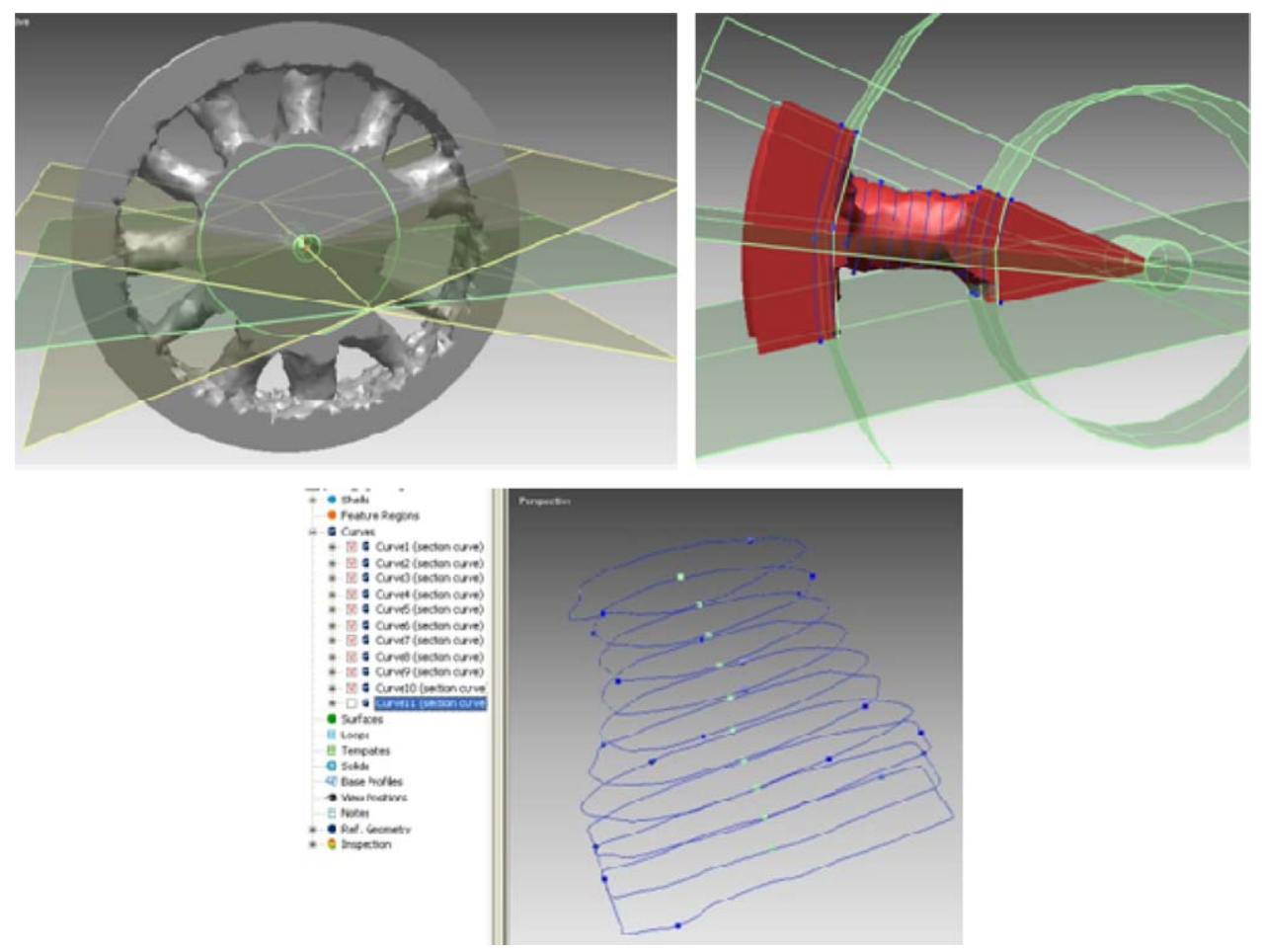

Fig. 10. Identification of reference geometries, extraction of profiles and calculation of respective centre of mass. 

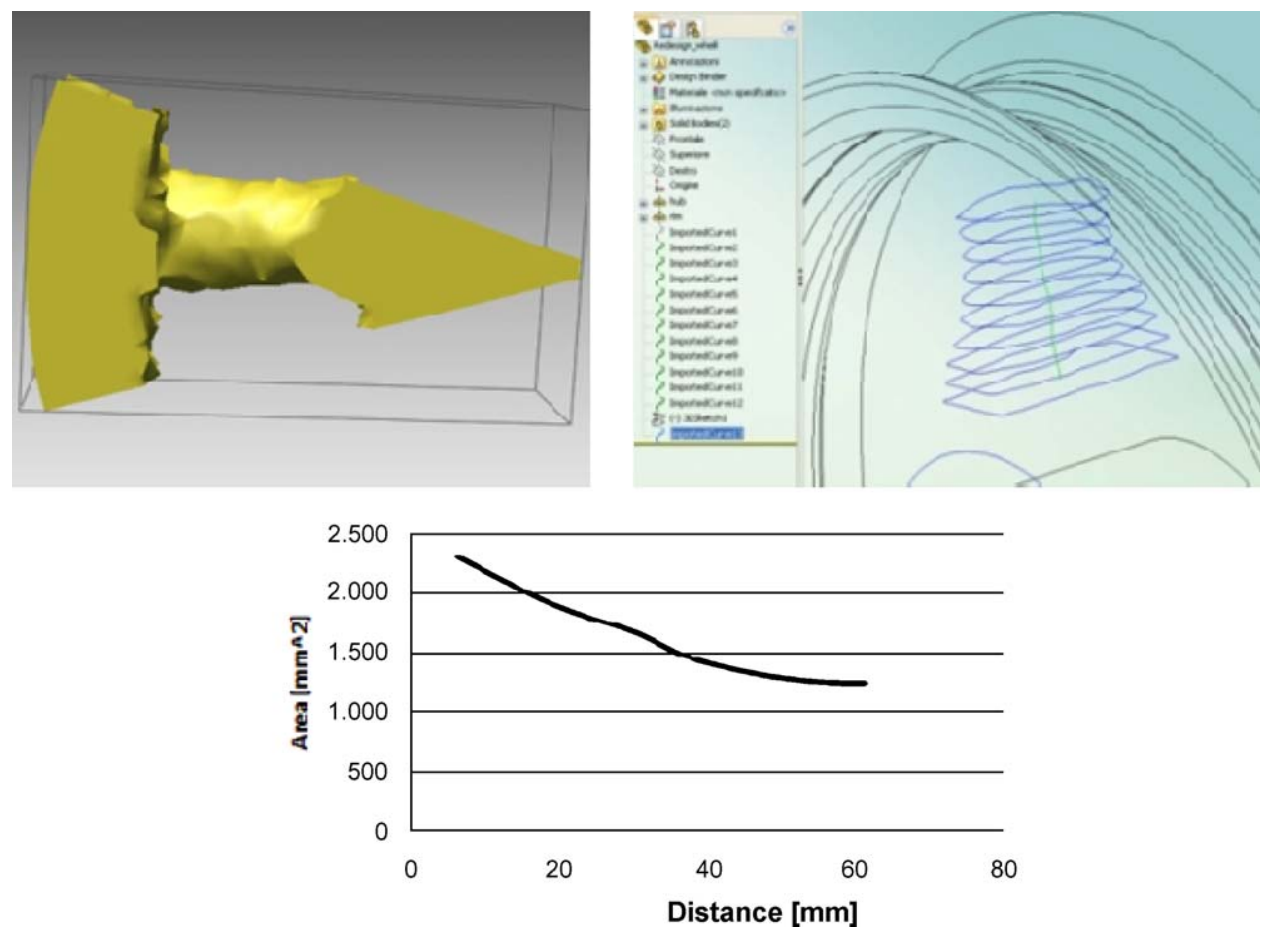

Fig. 11. Extraction of profiles and data analysis.

no longer as a unique entity, but by distinguishing invariant volumes from volumes to be optimized.

This procedure offers several advantages. It makes the data transfer from CAD to CAE environment much more effective because it simplifies the choice of various volumes to which one may apply different collectors (in CAE environment), and, most of all, it allows to preserve such knowledge throughout the optimization process. In this way, only the optimized volumes have to be modelled. The inconvenient connected to modelling approach that joins standardized parts, consists in the need to "assemble" a library of parametrical volumes, realized ad hoc for each typology of component to be optimized. Nevertheless, it is worth to underline that the parts to be modelled are created through simple geometries, since they usually represent "full" volumes that will have to be optimized.

At the end of the optimization process, it is necessary to translate the results into a feature-based model through the CAD system, usable for successive analysing phases. One of the main problems deals with the voxel model generated by OSs. The format used is usually an STL format, which holds a high number of triangles (e.g. the wheel in Fig. 9 is constituted by 32,000 triangles) with frequent errors on the triangles themselves as non-manifold face, redundant face, crossing face and unstable face. Besides, a simple cleaning and correction of STL files is not sufficient to guarantee a rational integration of OSs with the geometric modeller. In fact, the problem of recognising the modelling features on the optimized volume remains unsolved. The attempts to reconstruct the shape starting from feature recognition tools have not led to acceptable results. A more suitable solution may be the possibility to find a series of discrete elements on such volume and decide which of these elements to use during the successive modelling phase $[2,3]$.

Due to these critical issues, the proposed methodology suggests the realization of a second $\mathrm{KB}$ interface (exemplarily implemented in Rapid Form, using RapidForm API) able to:

- reduce the number of triangles, in order to allow an efficient import to CAD environments,
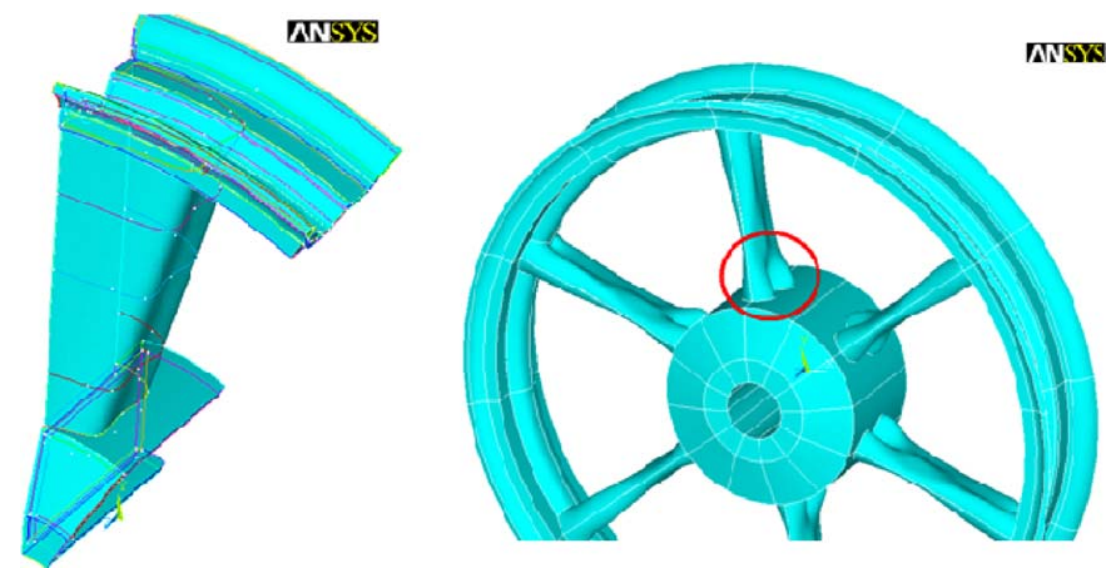

Fig. 12. Spline curves (design variables) of the spoke model subjected to shape optimization (left). Resulting shape-optimized wheel (right). 
- carry out checks to find and solve problems concerning faulty triangles,

- identify and extract, from optimized volumes, all geometrical elements considered to be useful during the successive modelling phase (planes, axes, points, profiles, trajectories, etc.).

Fig. 10 shows how a set of optimized spokes of a motorbike wheel were analyzed through RapidForm. This allowed identifying a series of radial profiles and a trajectory passing through the centre of gravity of the sections themselves (geometrical data useful for the designer during the modelling phase).

In order to increase the level of knowledge and extract further information (to be used during the redesign phase) from the optimized geometry, a geometrical analysis of the model may be particularly useful. In this way, it is possible to reconstruct the model shape in a less subjective way.

Fig. 11 shows the results of some analyses carried out on the above-mentioned spokes of the wheel. More specifically, it shows: the output of an optimization analysis; the profiles and the guidelines extracted from the optimized model; and finally the trend line of the area within the profiles according to their distance from the centre.

According to the proposed methodology, at the end of the optimization phase, the feature-based model can be obtained using two different typologies of data: invariant volumes (modelled as in the first phase of the process) and reference geometries extracted from the optimized volumes.

A third KB interface has been implemented to allow a completely automatic import of all data to a CAD environment. The availability of such data (invariant geometries, profiles and guidelines extracted from the geometry) noticeably simplifies the reconstruction phase. The KB interface allows to import curves and profiles both as 3D lines and as sketches.

\section{Exemplary application of the PROSIT algorithm: re-design of a scooter wheel}

This test case has been inspired by a real case study developed during a collaboration of the authors with the Italian motorbike

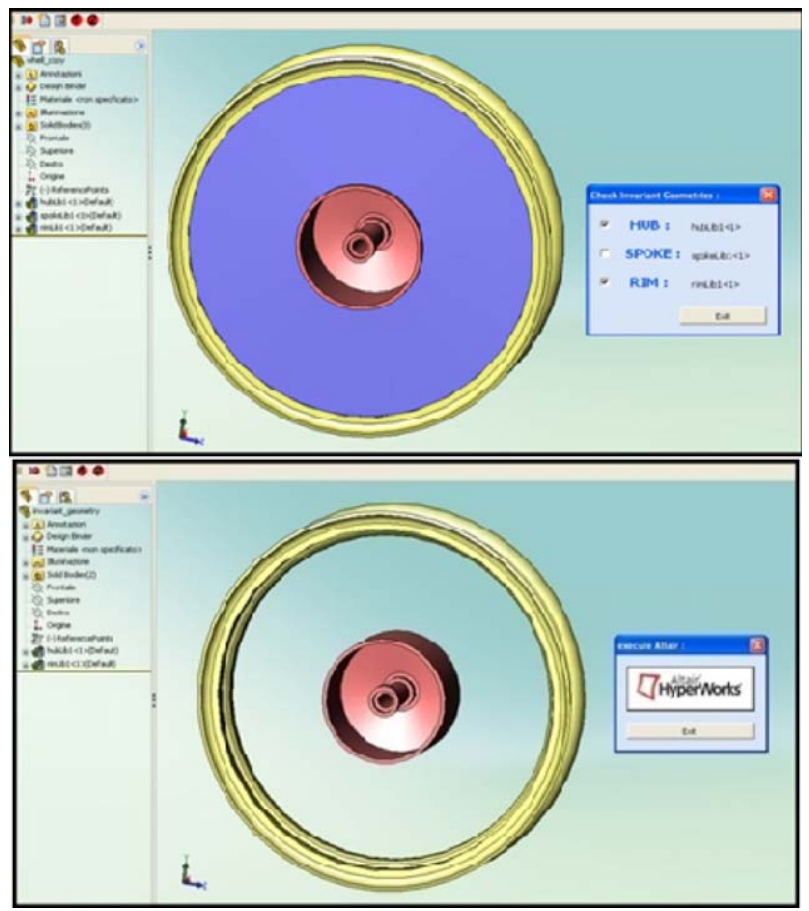

Fig. 13. Creating the model and managing information through the KB interface. producer Piaggio [23]. The goal of the project was the design of a plastic wheel for light moto-scooters mainly aimed at costs reduction, of course without compromising safety and mechanical performances. The traditional approach in Piaggio to assess the conformity of a wheel consists in three different experimental tests:

- deformation energy under high radial loads/displacements (simulating an impact against an obstacle);

- fatigue strength under rotary bending loads (simulating the operating conditions such as curves);

- fatigue strength under alternate torsional loads (simulating the accelerations and decelerations).

Preliminarily only a multi-objective optimization was performed by adopting these tests as reference criteria and assuming the presence of pre-defined set of spokes: the boundary conditions suggested the adoption of six spokes. The outline of the spoke has been modelled by two surfaces interpolating two set of spline curves (Fig. 12, left) whose control points represent the design variables, whereas the goal was minimizing the spoke volume (i.e. mass) under the constraint of a maximum Von Mises stress on the whole volume.

The obtained result shows a topological contradiction that is not solvable by a traditional design approach like this. The spokes, in fact, cannot be obtained with a simple technological process, like moulding, because they have a concave shape along the direction of mould extraction (Fig. 12, right).

Successively, to overcome this problem and obtain an innovative solution, the Prosit procedure has been implemented by using the same above-mentioned experimental tests as reference criteria for Design Optimization, under the constraint of manufacturability through die-moulding and the goal of minimizing mass since this parameter is directly related to costs.

Two functional surfaces were identified: the hub and the rim. The design domain for the topological optimization task coincides with the envelope of the volume of a classical wheel, while the internal side of the rim and the hub are assumed as invariant.

According to the proposed methodology, the first KB interface, exemplarily implemented as a SolidWorks plug-in, has been used to support, in an automatic way, the generation of the abovementioned volumes, through a dialogue window. For this test case,

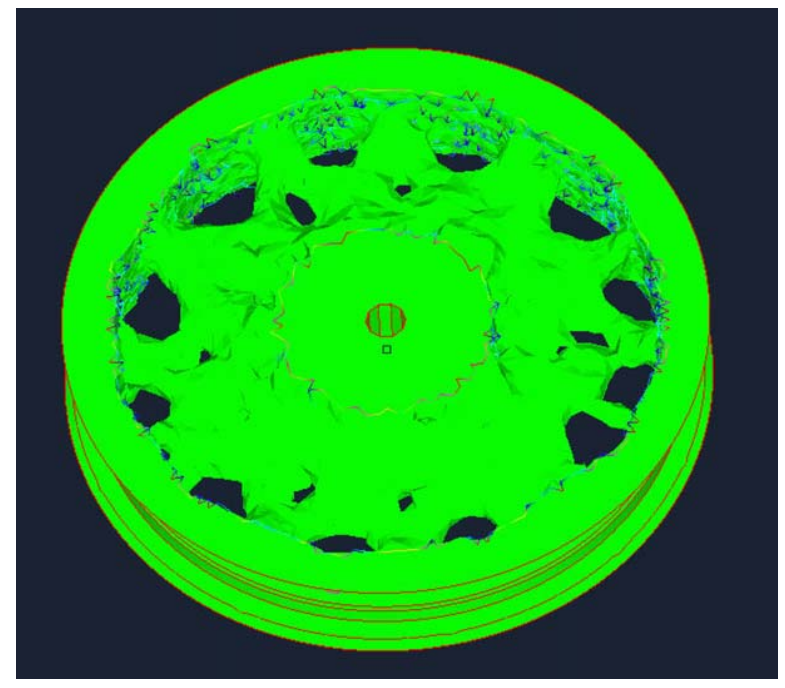

Fig. 14. Re-design of a scooter wheel: first step, multi-goal optimization (radial, bending, torsional loads are complementarily applied). The outputs of such optimization do not satisfy the whole set of product requirements. 
a specific DB related to the plastic moped wheel has been developed (Figs. 9 and 13).

The second step consisted in the definition of a multi-objective optimization problem, taking into account all the above-mentioned requirements (Fig. 14). Indeed, whatever is the weight assigned to each optimization goal, it is not possible to define a solution that fits with mass requirements (in the case of a plastic product mass and costs are directly related), mechanical performances, manufacturability.

Thus, three different mono-requirement topological optimizations related to the above-mentioned complementary tests have been defined. As a result, two different mass distributions were generated, the first related to the radial and torsional tests, the second to the rotary bending test (Fig. 15): more specifically, the first geometry suggests the creation of a flat web between the hub and the rim; while the second leads to a number of radial spokes with transversal ribs.

Before than facing such a topological contradiction, a further investigation must be done because the analysis revealed that the radial test by itself did not meet the expected requirements. When a mono-goal optimization does not converge to any acceptable solutions, the PROSIT guidelines suggest to split the problem further and to define a subset of optimization tasks with the same objective function, by removing the optimization constraints one-by-one.
As a result, the user is driven to the identification of design constraints in the following form: the constraint $\mathrm{X}$ should not be respected in order to fulfil the goal $\mathrm{G}$, but it should be respected in order to satisfy the requirement $\mathrm{R}$. In this case, the constraint to be removed in order to meet the design goal is the draw direction for manufacturability issues. In facts, the elimination of such a constraint leads to the geometry shown in Fig. 16: a hollow wheel with a double web supporting the side of the rim. Hence, the contradiction can be expressed in the form: "the wheel should present an axial draw direction in order to preserve manufacturability and should not present an axial draw direction in order to provide a sufficient radial stiffness".

The guidelines extracted from the TRIZ instruments for overcoming the geometrical contradictions suggest, among the others, the application of the segmentation principle (IP\#1). In other words, the design space can be divided in two parts, so that the manufacturability is preserved when the two parts are separated, while the stiffness satisfies the requirements when assembled. Fig. 17 shows the results of the topological optimization obtained by dividing the wheel into two halves.

It is worth noting that the web is characterized by a number of transversal ribs that remind the spokes suggested by the optimization under rotary bending loads (Fig. 15). Therefore, in this case, the solution of the contradiction within the radial load
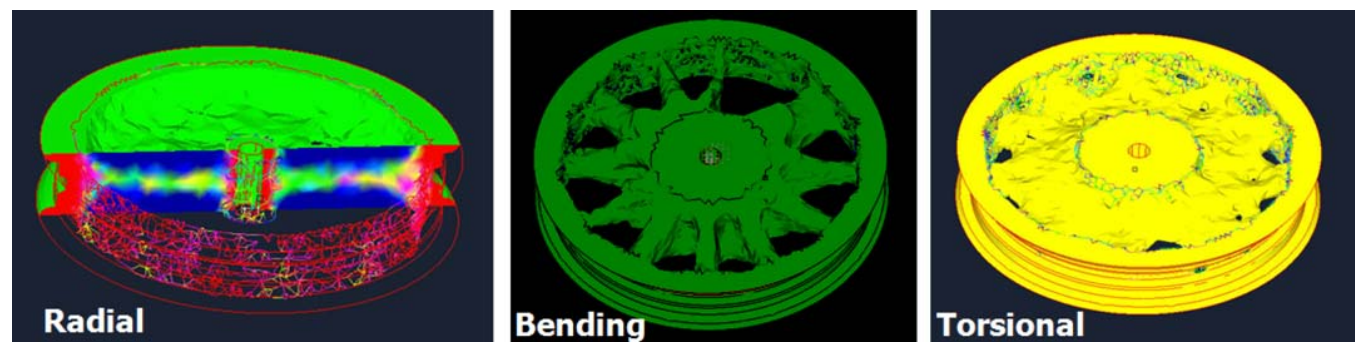

Fig. 15. Re-design of a scooter wheel: first step, comparison of the geometries arising from three complementary single-goal optimization tasks.
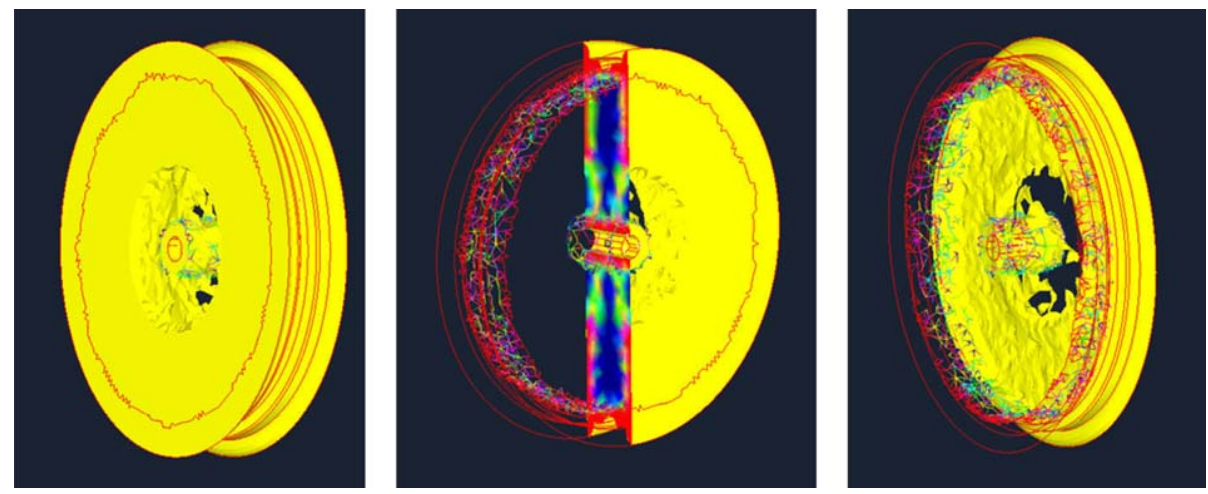

Fig. 16. Re-design of a scooter wheel: second step, in order to meet the design objectives, the manufacturability constraint should be removed.
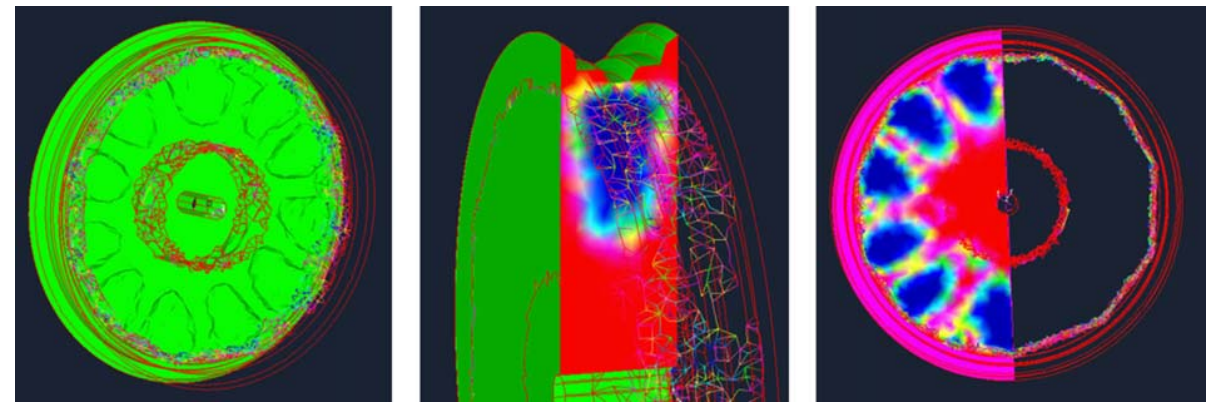

Fig. 17. Re-design of a scooter wheel: the geometrical contradiction can be overcome by applying the segmentation principle. 

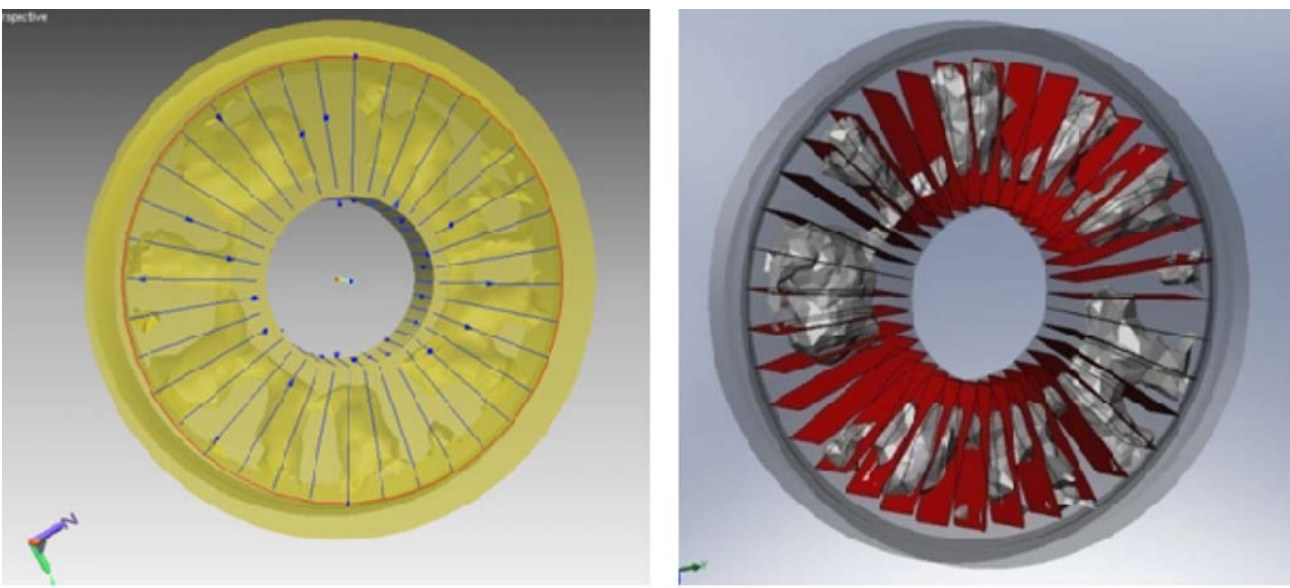

andamento area delle sezioni radiali:

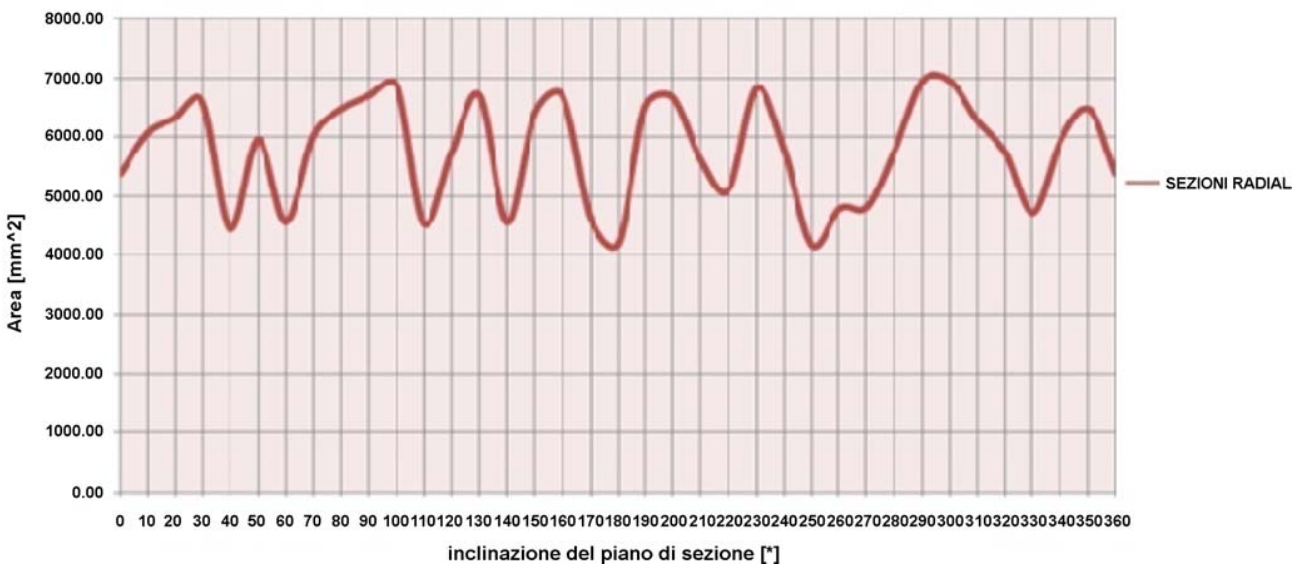

Fig. 18. Optimized single-waved-web wheel (above). Identification of 11 "waves" using the radial sections (below).

test brings to a geometry that fits with the results of the other mono-goal optimization tasks above defined and the overall process is converged.

Another suggestion provided by the PROSIT guidelines is to move from a flat web to a waved web (IP\#17: another dimension).

This conceptual result can be translated into a geometrical model to be defined through technological features according to the second main goal of the PROSIT project. In order to redesign the part and to obtain a feature-based manufacturable product model, the KB interface allows to import into the CAD environment both the invariant volumes and the data extracted from geometrical analysis on the optimized volume.

The following figures show the results of some analysis carried out on the optimized volume of the wheel. In details, Fig. 18 shows
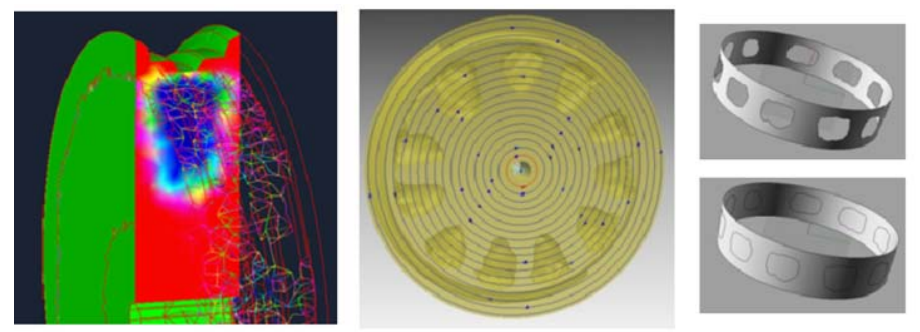

cylindrical sections

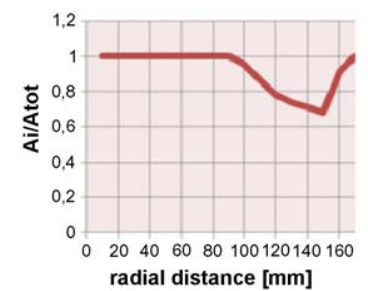

parallel sections
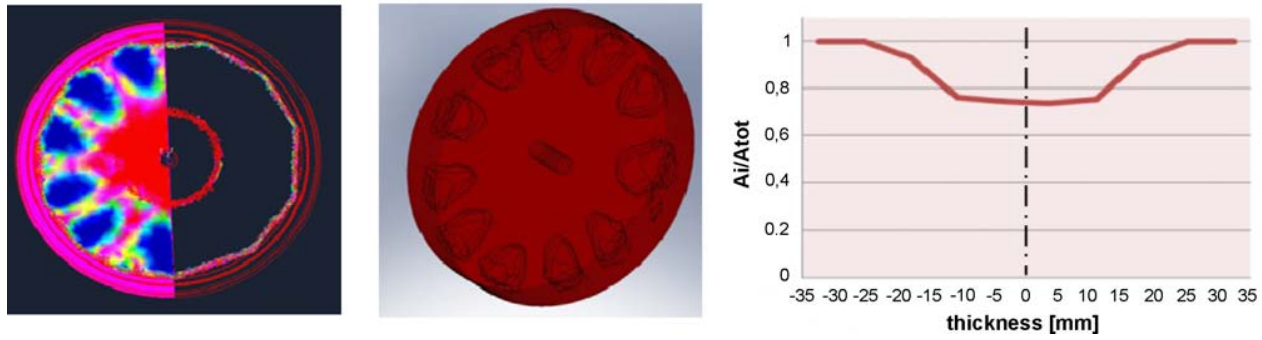

Fig. 19. Shape definition of the waved web. 

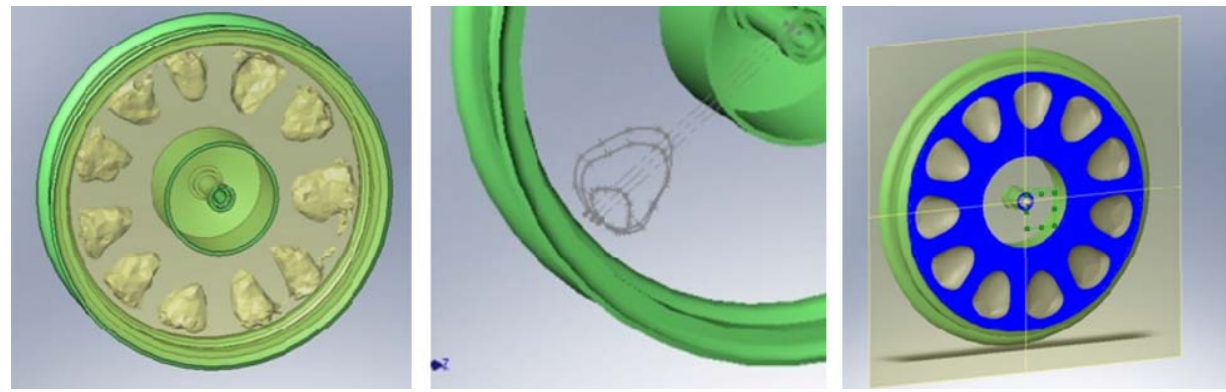

Fig. 20. Feature-based model of the wheel: invariant and optimized volumes; extraction of profiles; final result.

the definition of the number of the "internal" waves; the graph shows the area of the radial sections for different angles. Fig. 19 shows the definition of the shape of the waved web using both cylindrical and planar sections; the graphs show the ratio between the full and optimized section with respect to the section position. All these data are used to define the feature-based model of the wheel (see Fig. 20).

A standard FEM analysis of the final geometry revealed that a segmented-waved plastic wheel made with a resin PA6 with a $30 \%$ volume content of glass fibres (modulus of elasticity about $16 \mathrm{GPa}$ and stress to failure about $230 \mathrm{MPa}$ ) is capable to pass all the virtual tests required by Piaggio proprietary standards.

\section{Conclusions and future work}

The present paper has addressed the integration of ComputerAided Innovation systems, Optimization systems and PLM/EKM tools as a means to improve the innovation resources and the efficiency of a product development cycle. The rationale of this research is the lack of formalized and validated procedures allowing the systematic introduction and integration of these tools in the design process. The development of these integration guidelines, that is the most innovative aspect of the research, should provide advantages in terms of design costs reduction, errors reduction, product quality improvement, process execution time and more effective internal and external knowledge use and share.

A relevant aspect of the PROSIT project is the integration of apparently incompatible tools, thanks to the new role and way of usage of the Optimization systems. Moreover, in the mind of the authors, the methodology, through the definition of guidelines and best practices, can help overcoming classical interoperability problems affecting the design process, providing a common base for knowledge sharing and for a better interconnection between different systems and applications.

It is worth noticing that the PROSIT project does not aim at the creation of a fully automatic system for design embodiment, because both the comprehension of the root-cause of a geometrical contradiction and, most of all, the translation of the TRIZ principles into a new set of optimization tasks, requires a creative even if systematic step, demanded to the designer. Besides, the results obtained so far suggest the investigation of semi-automatic procedures to speed-up some routine tasks like the comparison of the outputs of the single-goal optimization tasks, as well as the removal of the optimization constraints oneby-one, when a single optimization does not converge to a solution.

As mentioned above, the proposed approach has been largely tested in several case studies in the field of machine design embodiment, thus when the functional architecture of the technical system is defined and the goal is the identification of a structure capable to behave accordingly. In this phase the designer mostly works with geometrical variables (related to topology, shape and size), and this justifies the focus on geometrical contradictions of the proposed methodology. It is worth to note that the obtained results show better performances than those obtained by a classical multi-goal optimization [24], thus confirming a contribution to the generation of "inventive" solutions also in the design embodiment phase.

Nevertheless, the idea behind the PROSIT project can be virtually extended to any phase/field of application where optimization software systems exist: in this case the accomplishment of different single-goal optimization tasks will produce general TRIZ contradictions (not necessarily involving the geometry) to be approached with classical TRIZ instruments. The authors have just started such an investigation in the field of process optimization. As a minimal preliminary result, the proposed methodology confirms to provide means to elicit hidden contradictions by means of a systematic process that requires minimal involvement by the designer.

Besides, further research still needs to be accomplished in order to automate the generation of new solutions: in facts, despite the promising preliminary results reported in [24], the designer still plays a crucial role, while the PROSIT guidelines should be considered just as a procedural support to his activity even in case of limited TRIZ knowledge.

In conclusions, the PROSIT approach is ready to be adopted as a framework for the integration of actually available Optimization and PLM systems. Among the proposed guidelines, those based on the TRIZ theory can be considered as a list of requirements for a novel CAI system especially suited for design embodiment.

\section{Acknowledgements}

The PROSIT project (Product development and systematic innovation http://www.kaemart.it/prosit) is co-funded by the Italian Ministry of University and Research.

The authors would like to thank M. Bordegoni and M. Bertoni from Politecnico di Milano and F. Rotini and F. Frillici from Università di Firenze, L. Barbieri and F. Bruno from Università della Calabria, and F. Cappello and T. Ingrassia from Università di Palermo for their contribution to the development of the PROSIT project.

\section{References}

[1] U. Cugini, G. Cascini, M. Ugolotti, Enhancing interoperability in the design process-the PROSIT approach, in: Proceedings of the 2nd IFIP Working Conference on Computer-Aided Innovation, Brighton (MI), USA, October 8-9, 2007 published on Trends in Computer-Aided Innovation, Springer, ISBN 978-0-38775455-0, pp. 189-200.

[2] L. Barbieri, F. Bruno, M. Muzzupappa, U. Cugini, Design automation tools as a support for knowledge management in topology optimization, in: Proceedings of the ASME 2008 International Design Engineering Technical Conferences \& 
Computers and Information in Engineering Conference (IDETC/CIE 2008), Brooklyn, New York, USA, August 3-6, 2008.

[3] L. Barbieri, F. Bruno, M. Muzzupappa, U. Cugini, Guidelines for an efficient integration of topological optimization tools in the product development process, in: Third International Conference on Design Computing and Cognition, Atlanta, USA, June 23-25, 2008.

[4] G. Cascini, P. Rissone, F. Rotini, From design optimization systems to geometrical contradictions, in: Proceedings of the 7th ETRIA TRIZ Future Conference, Frankfurt, Germany, November 6-8, 2007.

[5] G. Cascini:, State-of-the-Art and trends of Computer-Aided Innovation tools Towards the integration within the Product Development Cycle, Building the Information Society, Kluwer Academic Publishers, 2004, pp. 461-470 (ISBN 14020-8156-1).

[6] H.T. Chang, J.L. Chen, The conflict-problem-solving CAD software integrating TRIZ into eco-innovation, Advances in Engineering Software 35 (2004) 553566.

[7] N. Leon, J.M. Cueva, J. Gutiérrez, D. Silva, Automatic shape variations in 3D CAD environments, in: Proceedings of the 1st IFIP TC5 Working Conference on Computer Aided Innovation, Ulm, Germany, November 14-15, 2005 (ISBN 3-00017325-0).

[8] A. Albers, N. Leon, H. Aguayo, T. Maier, Comparison of strategies for the optimization/innovation of crankshaft balance, in: Proceedings of the 2nd IFIP Working Conference on Computer Aided Innovation, Brighton (MI), USA, October 8-9, 2007, published on Trends in Computer-Aided Innovation, Springer, ISBN 978-0387-75455-0.

[9] N. Khomenko, R. De Guio, L. Lelait, I. Kaikov, A framework for OTSM-TRIZ based computer support to be used in complex problem management, International Journal of Computer Application in Technology (IJCAT) 30 (1/2) (2007).

[10] S. Szykman, S.J. Fenvesa, W. Keirouzb, S.B. Shooter, A foundation for interoperability in next-generation product development systems, Computer-Aided Design 33 (June (7)) (2001) 545-559.

[11] F. Mervyn, A. Senthil Kumar, S.H. Bok, A.Y.C. Nee, Developing distributed applications for integrated product and process design, Computer-Aided Design 36 (July (8)) (2004) 679-689

[12] K. Saitou, K. Izui, S. Nishiwaki, P. Papalambros, A survey of structural optimization in mechanical product development, Journal of Computing and Information Science in Engineering 5 (September (3)) (2005) 214-226.

[13] R. Kicinger, T. Arciszewski, K. De Jong, Evolutionary computation and structural design: a survey of the state-of-the-art, Computers and Structures 83 (2005) 1943-1978.

[14] D. Spath, W. Neithardt, C. Bangert, Optimized design with topology and shape optimization, Proceedings of the Institution of Mechanical Engineers Part B: Journal of Engineering Manufacture 216 (8) (2002) 1187-1191.

[15] D.W. Rosen, I..R. Grosse, A feature based shape optimisation technique for the configuration and parametric design of flat plates, Engineering with Computers 8 (1992) 81-91.

[16] A.R. Yildiz, N. Ozturk, N. Kaya, F. Ozturk, Integrated optimal topology design and shape optimisation using neural networks, Structural and Multidisciplinary Optimisation 25 (4) (2003) 251-260.

[17] N. Ozturk, A.R. Yıldız, N. Kaya, F. Ozturk, Neuro-genetic design optimisation framework to support the integrated robust design optimisation process in CE, Concurrent Engineering: Research and Applications 14 (1) (2006) $5-16$.

[18] Z. Huang, D. Yip-Hoi, High-level feature recognition using feature relationship graphs, Computer-Aided Design 34 (2002) 561-582.

[19] H.L. Lockett, M.D. Guenov, Graph-based feature recognition for injection moulding based on a mid-surface approach, Computer-Aided Design 37 (2005) 251262.

[20] E. Brousseau, S. Dimov, R. Setchi, Knowledge acquisition techniques for feature recognition in CAD models, Journal of Intelligent Manufacturing 19 (2008) $21-32$.

[21] I.L. Vikentiev, V.I. Yefremov, Index of Geometric Effects, first published in the collection "Rules of a Game Without Rules", Petrozavodsk, Karelia, 1989, ISBN 57545-0108-0 (in Russian).

[22] I.L. Vikentiev, Do we really understand geometry? http://www.triz-chance.ru/ geometry_en.html.

[23] G. Cascini, P. Rissone, Plastics design: integrating TRIZ creativity and semantic knowledge portals, Journal of Engineering Design 15 (August (4)) (2004) 405-424 (Special Issue: Knowledge Engineering \& Management Issues in Engineering Design Practices).

[24] G. Cascini, U. Cugini, F. Frillici, F. Rotini, Computer-Aided Conceptual Design through TRIZ-based density manipulation, in: Proceedings of the 19th CIRP Design Conference, Special session on Systematic Processes for Creative and Inventive Design, Cranfield University, UK, March 30-31, 2009.

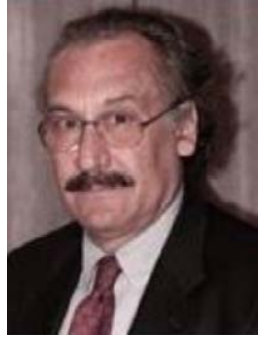

Umberto Cuguni holds a Degree in Mechanical Engineering from Politecnico di Milano and Master degree in Management and Business Administration from Università Bocconi. He is currently Full Professor of Machine Design, CAD and Virtual Prototyping, Faculty of Industrial Engineering, Politecnico di Milano. He is currently Deputy coordinator of the European project FP6-IST SATIN, Coordinator of the MUR-FIRBMade in Italy project and Chairman of IFIP WG 5.2 (Computer-Aided Design) since August 2000. He is author of more than 200 scientific papers published in national/international journals and conference proceedings. He is Member of the Editorial Board of "International Journal for Manufacturing Science \& Production" and Member of the Board of Directors of Fondazione Politecnico. He has been Coordinator of the PhD program on "Virtual Prototypes and Real Products", Politecnico di Milano from XVII edition; President of Consorzio Politecnico Innovazione; Coordinator of the European project FP6-IST Touch and Design (T'nD); Director of Progetto Finalizzato Robotica - CNR, 19881996 and Coordinator of the PhD program on Industrial Production Enginering Univ. Parma, VIII-XVI editions.

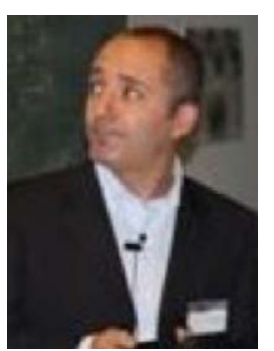

Gaetano Cascini took a Master degree with honours in Mechanical Engineering and a PhD in Machine Design at the University of Florence. He is certified 4th level TRIZ specialist by MA TRIZ (the international TRIZ Association, certificate N.80). He has been a researcher at the University of Florence from 1999 to 2008. He is currently: Associate Professor at Politecnico di Milano, Faculty of Industrial Engineering, President of ETRIA (European TRIZ Association) and Vice-chair of the "Computer-Aided Innovation" workgroup of IFIP (International Federation for Information Processing). His research interests include: methods and tools for inventive design (scientific formalization and evolution of methods for inventive problem solving, modelling and analysis of the product cycle and Business Process Reengineering through process innovation, Knowledge Management and text mining algorithms for patent analysis) and Computer-Aided systems (development of Knowledge-Based CAD tools and novel techniques of topological optimization). Dr Cascini is author of more than 70 papers presented at International Conferences and published in authoritative Journals and 8 patents.

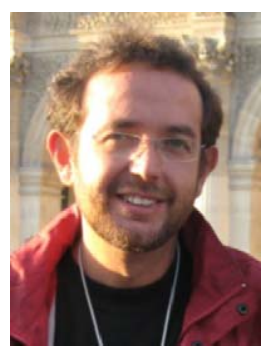

Maurizio Muzzupappa is an Associate professor of Computer-Aided Design at the Department of Mechanical Engineering of the University of Calabria. From 1989 to 1992 , he frequented his PhD at the Department of Mechanical Engineering of the University of Pisa. His current research activities include: applications of Virtual and Augmented Reality (VR\&AR) to the design process of the industrial products (specific topics are the usability of the user interface). Other topics are: concurrent engineering (in particular, the computer support of cooperating virtual engineering teams for design review), CAD automation and reverse engineering.

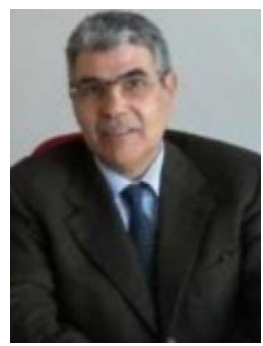

Vincenzo Nigrelli is Full professor of Machine Drawing at the Department of "Meccanica", University of Palermo and Head of the Department, since November 2008. Professor Nigrelli is author of more than 100 papers in the following such fields as: reverse engineering, rapid prototyping \& tooling, CAD, topological and shape optimization, image processing, nondestructive evaluation, characterization of materials, experimental mechanics, stress analysis, fracture mechanics. He is active in national research programs, research tutor and team leader of the project "NonContact Ultrasonic System for Rail Track Inspection" financed by the European Community under the 6th Framework Research Programme. 